\title{
Información Contable en la Predicción de Insolvencia: estudio inferencial univariante aplicado a empresas españolas
}

Data in Prediction of Insolvency: inferential univariate study applied to Spanish firms

\section{Romualdo Douglas Colauto}

Doutorado em Engenharia de Produção na Universidade Federal de Santa Catarina

Professor do Programa Programa de Mestrado em Ciências Contábeis da Universidade Federal de Minas Gerais

Endereço: Departamento de Ciências Contábeis. Av. Antônio Carlos, 6.627 - Faculdade de Ciências Econômicas - sala 2042 - Pampulha

CEP: 31270-901 - Belo Horizonte/MG - Brasil

E-mail: rdcolauto@terra.com.br

Telefone: (31) 3409-7056

\section{Laura Edith Taboada Pinheiro}

Doutorado em Contabilidade e Finanças pela Universidad de Zaragoza - Espanha

Professora da Universidade Federal de Minas Gerais

Endereço: Departamento de Ciências Contábeis. Av. Antônio Carlos, 6.627 - Faculdade de Ciências Econômicas - sala 2038 - Pampulha

CEP: 31270-901 - Belo Horizonte/MG - Brasil

E-mail: 1taboada@face.ufmg.br

Telefone: (31) 3409-7271

\section{Juliano Lima Pinheiro}

Doutorado em Contabilidade e Finanças pela Universidad de Zaragoza - Espanha

Professor da Faculdade de Ciências Humanas de Pedro Leopoldo

Endereço: Rua Teófilo Calazans de Barros, 100 - Santo Antônio da Barra

CEP: 33600-000 - Pedro Leopoldo/ MG - Brasil

E-mail: jlp@gold.com.br

Telefone: (31) 3662-4000

Artigo recebido em novembro de 2008. Passou por uma avaliação double blind review em setembro de 2009. Aceito em novembro de 2009 pela Editora Científica Sandra Rolim Ensslin. 


\title{
Resumen
}

El objetivo de este trabajo es realizar estimaciones que permitan establecer relaciones significativas entre determinados ratios financieros y la insolvencia empresarial. La muestra envuelve empresas españolas que cotizan en Bolsa no pertenecientes al sector financiero y de seguros. Los resultados indican que varios ratios contables consiguen clasificar las empresas en solventes e insolventes con un grado de acierto del 95\% para el año anterior al fracaso. Los indicadores de Rentabilidad del Activo, Margen de Beneficio del Resultado Ordinario y Cobertura de Gastos Financieros fueron los que resultaron con mayor poder discriminante. Se concluye, por lo tanto, que modelos univariantes pueden presentar expresiva capacidad predictiva del riesgo de insolvencia, además de contar con mayor practicidad en su proceso de estimación e implementación, en contraposición a los modelos multivariantes.

Palabras-Clave: Fracaso Empresarial, Insolvencia, Predicción de Quiebras, Índices Financieros.

\begin{abstract}
The objective of this study is to make estimations which allow the establishment of significant relationships between determined financial ratios and company insolvency. The sample was taken from open Spanish companies which do not pertain to the financial or insurance sector. The results show that various accountancy ratios are able to classify companies as solvent or insolvent with a $95 \%$ degree of certainty when taken from the previous year to failure. The Rate of Return on Assets, Profit Margin from the Operating Result and Cover for Financial Expenses were those indicators which provided the strongest discriminant power. It was therefore concluded that univaried models show significant capacity to predict the risk of insolvency. Besides that, these models count on greater practicability in the process of estimation and implementation, in contrast to the multivaried models.
\end{abstract}

Key-words: Business Failure, Insolvency, Prediction of Bankruptcy, Financial Ratios.

\section{Introducción}

Debido a la gran cantidad de causas que pueden llevar a la discontinuidad de la actividad de una empresa, las cuales no siempre están presentes en todo fracaso empresarial, y a la falta de conocimiento de cómo relacionar éstas con la posterior insolvencia de la empresa, aún no se ha llegado a establecer una teoría general del fracaso empresarial, que permita alertar con antecedencia sobre el mismo, de modo a evitar las indeseables consecuencias que conlleva.

En este sentido, la anticipación del fracaso empresarial viene siendo objeto de numerosos estudios a fin de permitir que todos aquellos relacionados económicamente con la empresa cuenten con herramientas que permitan detectar la insolvencia con antecedencia.

Onusic y Casa Nova (2006) destacan que los modelos de predicción de insolvencia son de grande auxilio en la evaluación del riesgo, siendo utilizados como una herramienta importante en el análisis de desempeño de las empresas y apoyo a las decisiones de crédito. 
Ontiveros y Valero (1987, p. 25) denominan a los modelos de predicción de crisis empresariales "modelos microeconómicos", dado que éstos parten de la base de que los principales problemas del fracaso empresarial se localizan en la misma empresa, de forma que se tratará, en definitiva, de determinar los rasgos más significativos que pueden observarse en las empresas en crisis, por contraposición con las empresas con éxito, rasgos que sirvan para detectar, e incluso predecir, la situación de fracaso.

En los últimos años fueron desarrollados varios modelos de previsión de insolvencia aplicables a la realidad de empresas de diversos países, sectores y tamaños y por medio de diversificadas técnicas, no obstante, en función de las limitaciones que estos presentan, se hace necesario continuar los estudios sobre modelos aplicables a otras realidades.

Este trabajo tiene por objetivo realizar estimaciones que permitan establecer relaciones significativas entre determinados ratios financieros y la insolvencia empresarial, centrando el mismo en un ámbito concreto de la realidad empresarial española, el cual corresponde a empresas industriales de gran dimensión que cotizan en bolsa.

La estructura del artículo se ha desarrollado como se describe a continuación. Tras esta introducción, el segundo apartado se dedica a la revisión de la literatura sobre modelos de predicción de insolvencia empresarial. A continuación, en el tercero apartado, se expone el diseño del estudio empírico realizado, que incluye la metodología aplicada, la descripción de la muestra y las variables consideradas. En el cuarto apartado se presentan los resultados alcanzados y su análisis. En el quinto apartado se realiza una breve comparación con los resultados de otros estudios y, finalmente, en el sexto y último apartado se exponen las conclusiones.

\section{Estudios previos sobre modelos univariantes de predicción de fracaso empresarial}

El estudio más clásico, basado en ratios contables, es el de Beaver (1966) que, conforme coloca Martín (1997, p.40), a pesar de su antigüedad, conserva todavía su vigencia metodológica. El objetivo del estudio de Beaver (1966) es la predicción de la insolvencia empresarial a través de ratios, concluyendo que el ratio Cash flow a Deuda total es el de mayor valor predictivo. Las técnicas utilizadas fueron la comparación de las medias para obtener un perfil de las empresas fracasadas y no fracasadas en los años anteriores al fracaso y el test de clasificación dicotómica, que se basa en un único ratio y representa, a diferencia del anterior, un test predictivo.

Deakin (1972) busca proporcionar un modelo alternativo al de Beaver 
(1966) aplicando nuevamente el análisis dicotómico y acrecentando también el análisis discriminante lineal múltiple, obteniendo resultados próximos con el análisis dicotómico y alta precisión en los resultados con el análisis discriminante. Por su parte, Wilcox (1973) se plantea el problema de encontrar una explicación al poco poder discriminante que tienen algunos ratios, en especial los referentes a circulantes. Elabora un modelo que utiliza instrumentos estadísticos y que simula un juego, siendo su principal logro el encontrar un modelo teórico con los resultados obtenidos por otros autores el cual puede ser aplicado empíricamente. Un resumen de los estudios comentados hasta aquí puede observarse a través del Cuadro 1.

Cuadro 1: Investigaciones empíricas más relevantes sobre modelos de predicción univariantes de insolvencia de empresas industriales

\begin{tabular}{|c|c|c|c|c|c|c|c|}
\hline Autor & $\begin{array}{l}\text { Comp. } \\
\text { Muestra } \\
\text { (F y S) }\end{array}$ & Sectores & Emparej. & Período & $\begin{array}{c}\text { Ratios } \\
\text { seleccio- } \\
\text { nados }\end{array}$ & Técnica Estad. & $\begin{array}{l}\text { Rtdos. M. } \\
\text { Est. }\end{array}$ \\
\hline $\begin{array}{l}\text { Beaver } \\
(1966)\end{array}$ & $\begin{array}{l}79 \text { y } 79 \text { (de } \\
\text { Estados } \\
\text { Unidos) }\end{array}$ & $\begin{array}{l}38 \text { sectores } \\
\text { industriales } \\
\text { diferentes }\end{array}$ & $\begin{array}{l}\text { Tamaño de } \\
\text { activo y } \\
\text { tipo de } \\
\text { industria }\end{array}$ & $\begin{array}{l}1954- \\
1964\end{array}$ & $\begin{array}{l}\text { Cash } \\
\text { Flow/ } \\
\text { Deuda } \\
\text { Total } \\
\text { (CF/DT) } \\
\text { e Benef. } \\
\text { Neto/ } \\
\text { Activo } \\
\text { Total } \\
\text { (BN/AT) }\end{array}$ & Clasif.Dicotómica & $\begin{array}{l}\mathrm{n}-1: \\
\text { CF/DT: } 90 \% \\
\text { BN/AT: } 88 \% \\
\text { n-2: } \\
\text { CF/DT: } 82 \% \\
\text { BN/AT: } 85 \%\end{array}$ \\
\hline $\begin{array}{l}\text { Deakin } \\
(1972)\end{array}$ & $\begin{array}{l}32 \text { y } 32 \text { de } \\
\text { estimación } \\
\text { (de Estados } \\
\text { Unidos) }\end{array}$ & Industriales & $\begin{array}{l}\text { Tamaño de } \\
\text { activo, tipo } \\
\text { de industria } \\
\text { y año }\end{array}$ & $\begin{array}{l}1964- \\
1970\end{array}$ & $\begin{array}{l}\text { Aplica } \\
\text { los } 14 \\
\text { ratios de } \\
\text { Beaver } \\
\text { con } \\
\text { mejores } \\
\text { rtdos. }\end{array}$ & $\begin{array}{l}\text { Clasif. Dicotómica } \\
\text { (y también el } \\
\text { Análisis } \\
\text { Discriminante) }\end{array}$ & $\begin{array}{l}\text { CF/DT es el } \\
\text { que presenta } \\
\text { mayor poder } \\
\text { de clasif. } \\
\text { n-1: } 80 \% \text { y } \\
\text { n-2: } 84 \%\end{array}$ \\
\hline $\begin{array}{l}\text { Wilcox } \\
\text { (1973) }\end{array}$ & $\begin{array}{l}52 \text { y } 52 \text { (de } \\
\text { Estados } \\
\text { Unidos) }\end{array}$ & Industriales & $\begin{array}{l}\text { Tamaño de } \\
\text { activo, tipo } \\
\text { de industria } \\
\text { y año }\end{array}$ & $\begin{array}{l}1955- \\
1971\end{array}$ & $\begin{array}{l}\text { Mejores } \\
\text { ratios de } \\
\text { Beaver }\end{array}$ & Cadenas de Markov & $\begin{array}{l}\text { Mejores } \\
\text { rtdos. que } \\
\text { Beaver } \\
\text { n-1: } 94 \% \\
\text { n-2: } 90 \%\end{array}$ \\
\hline Lev (1973) & $\begin{array}{l}\text { Igual } \\
\text { muestra } \\
\text { Beaver }\end{array}$ & $\begin{array}{l}\text { Igual muestra } \\
\text { Beaver }\end{array}$ & $\begin{array}{l}\text { Igual } \\
\text { muestra } \\
\text { Beaver }\end{array}$ & $\begin{array}{l}\text { Igual } \\
\text { muestra } \\
\text { Beaver }\end{array}$ & $\begin{array}{l}\text { Cash } \\
\text { Flow/ } \\
\text { Deuda } \\
\text { Total } \\
\text { (CF/DT) }\end{array}$ & $\begin{array}{l}\text { Anál. p/descompos. } \\
\text { basa-da en la } \\
\text { compar.de dos } \\
\text { masas } \\
\text { patrimoniales }\end{array}$ & $\begin{array}{l}\text { Ratio CF/DT } \\
\text { como el de } \\
\text { mayor poder } \\
\text { discriminante }\end{array}$ \\
\hline $\begin{array}{l}\text { Moyer } \\
(1977)\end{array}$ & $\begin{array}{l}\text { Igual } \\
\text { muestra } \\
\text { Beaver }\end{array}$ & $\begin{array}{l}\text { Igual muestra } \\
\text { Beaver }\end{array}$ & $\begin{array}{l}\text { Igual } \\
\text { muestra } \\
\text { Beaver }\end{array}$ & $\begin{array}{l}\text { Igual } \\
\text { muestra } \\
\text { Beaver }\end{array}$ & $\begin{array}{l}\text { Cash } \\
\text { Flow/ } \\
\text { Deuda } \\
\text { Total } \\
\text { (CF/DT) }\end{array}$ & $\begin{array}{l}\text { Anál. p/descompos. } \\
\text { basa-da en la } \\
\text { compar.de dos } \\
\text { masas } \\
\text { patrimoniales }\end{array}$ & $\begin{array}{l}\text { Ratio CF/DT } \\
\text { como el de } \\
\text { mayor poder } \\
\text { discriminante }\end{array}$ \\
\hline
\end{tabular}

Fonte: Elaborado por los autores.

Otros autores como Lev (1973) y Moyer (1977) aplicaron la técnica de análisis por descomposición, basada en la comparación de las distintas masas patrimoniales, a la misma muestra de Beaver (1966). Como expone Lev (1978, p. 176), las medidas de descomposición indican la estabilidad relativa de la división por conceptos en los estados financieros, pudiendo esperar que las empresas 
que fracasan tendrán, cambios importantes en sus activos y pasivos, lo cual fue confirmado en su estudio.

\section{Metodología}

La presente investigación se caracteriza como cuantitativa y descriptiva, utilizándose técnicas y métodos para la obtención, tratamiento e interpretación de datos de forma a concluir para la población objeto de estudio los resultados obtenidos a partir de los datos de una muestra de la población.

Para alcanzar el objetivo propuesto se parte de un análisis descriptivo sobre las características económico-financieras de las empresas que fracasan, frente a las que no lo hacen. Seguidamente, y con el fin de identificar un perfil económico financiero de ambos grupos de empresas a partir de las cuentas anuales, se aplican técnicas estadísticas que permitan identificar qué variables diferencian o clasifican mejor a ambos grupos de empresas y permitan avalar la separación previa de la muestra en los dos grupos de empresas.

La metodología utilizada para realizar el estudio descriptivo del comportamiento de ambos grupos de empresas fue a través de instrumentos de análisis exploratorio, tales como: medidas de tendencia central (media y mediana); medidas de dispersión (desviación típica, coeficiente de variación, mínimos y máximos); y medidas de distribución (asimetría y curtosis o apuntamiento).

Seguidamente, se profundiza en la investigación empírica a través del proceso inferencial univariante para identificar y definir las variables que individualmente consideradas permitan explicar y predecir la insolvencia empresarial. Beaver (1966) fue el precursor en la aplicación de la técnica univariante para la estimación de modelos de predicción de insolvencia y lo realizó por medio del Análisis Dicotómico, el cual consiste en ordenar todas las observaciones de la muestra por cada ratio analizado y encontrar un punto de corte que separe a las empresas insolventes de las solventes de forma tal que se minimice el número de observaciones incorrectas. Posteriormente, autores como Deakin (1972) y Elam (1975) también aplicaron la técnica de Análisis Dicotómico empleada por Beaver (1966) como parte de sus investigaciones, a los cuales les siguieron otros autores, destacándose en España los estudios de Lizarraga (1997), García et al (1998) y Somoza (2000).

Primeramente, se identifican los ratios con diferentes medias entre los grupos a través de la técnica Análisis de la Varianza de un factor (ANOVA), a fin de conocer los posibles ratios con poder discriminante. Seguidamente se realiza el Análisis de Perfiles de Beaver (1966) en base a los resultados del ANOVA a fin de definir un perfil económico-financiero que muestre las diferencias, estadísticamente significativas, entre los dos colectivos estudiados, a fin de corroborar 
las hipótesis que surgieron del análisis descriptivo.

El Análisis de Perfiles de Beaver (1966), es una representación de cada uno de los ratios para cada estado de la empresa, fracasada o sana, y es aplicado en cada año anterior al fracaso. Éste permite analizar la diferencia entre ambos tipos de empresas teniendo en cuenta sólo la media, no así la dispersión, no proporcionando una idea sobre la magnitud o cuantificación de dichas diferencias.

Finalmente, se seleccionan los mejores modelos predictivos univariantes a través del Análisis Dicotómico de Beaver (1966). La prueba de clasificación dicotómica de Beaver (1966) es un test predictivo, cuya finalidad última es seleccionar que ratio permite una mejor discriminación entre ambos grupos de empresas. Esta prueba se aplica a cada año anterior a la insolvencia. El punto de corte se determina mediante un proceso de prueba y error, por el cual se van fijando diferentes valores y se va tanteando cuál de ellos produce menores errores de clasificación, de tal forma que si:

Ratio $_{i} \leq$ punto de corte $=$ clasificación: fracasada

Ratio $_{i}>$ punto de corte $=$ clasificación: sana

Por tanto, para cada ratio se determina el punto de corte que separa a las empresas fracasadas de las sanas, observándose para cada uno el porcentaje de acierto y de error en la clasificación de ambos grupos de empresas. Los Tipos de Error ante una clasificación dicotómica pueden ser:

Error Tipo I: clasificar una empresa fallida como sana

Error Tipo II: clasificar una empresa sana como fallida

Las consecuencias de incurrir en un tipo u otro de error son claramente distintas, así por ejemplo en el caso de concederse un préstamo o decidir invertir en una empresa, el coste de error Tipo I es mucho mayor que el Tipo II, puesto que el Tipo II es un coste de oportunidad asociado a la no elección de dicha empresa; en cuanto el Tipo I involucra la pérdida de parte o totalidad del capital invertido.

Por ello, dependiendo del caso, resulta aconsejable intentar minimizar el porcentaje de error Tipo I o Tipo II, en vez del porcentaje de error total. Por lo que en el análisis de la solvencia debería intentarse reducir el error Tipo I, ya que éste provoca consecuencias más serias que el Tipo II.

Para la selección de los mejores modelos univariantes, se parte de los ratios con mejor porcentaje de acierto en la clasificación de los grupos de empresas resultante del Análisis Dicotómico, analizándose luego las correlaciones, a través del coeficiente de correlación de Pearson, a fin de evaluar la dependencia entre cada una de las variables consideradas. Para la aplicación de las técnicas estadísticas mencionadas se utilizaron las herramientas informáticas de hoja de cálculo Excel y el paquete estadístico SPSS (Statistical Package of Social Sciences). 


\subsection{Descripción de la muestra}

Las empresas objeto del estudio son empresas españolas cotizadas en Bolsa no pertenecientes al sector financiero ni de seguros. El criterio de fracaso utilizado en este estudio fue el correspondiente a la delimitación legal, por el cual se consideran fracasadas aquellas empresas en las que se ha admitido la solicitud de Suspensión de Pagos o Quiebra.

De acuerdo con Gabás (1997, p. 15) la variedad de situaciones por las que puede transitar una empresa insolvente dificulta fuertemente dar el concepto de fracaso empresarial, lo que obliga a los investigadores de la insolvencia o del fracaso empresarial a definir su concepto propio de forma explícita, por lo que se utilizan variadas definiciones en función de los objetivos o en razón de la disponibilidad de datos.

Las empresas fracasadas fueron identificada a partir de la lectura de los hechos relevantes comunicados a la Comisión Nacional del Mercado de Valores en el período 1992 a 2001, de cada una de las 691 empresas no financieras ni de seguros supervisadas por la Comisión que comprendían la muestra total. De esta forma se identificaron 30 empresas fracasadas. Por ser necesario para la elaboración de modelos de predicción de insolvencia, se procedió a realizar, al igual que autores como Beaver (1966), Wilcox (1973), Altman (1968), Deakin (1972), Edminster (1972), Blum (1974), Taffler (1974) descrito en Taffler (1982), Taffler y Tisshaw (1977), Altman et al (1977), Gentry et al (1985), Lizarraga (1997), García (Coord.) (1997), Gallego et al (1997), Ferrando y Blanco (1998), entre otros, el emparejamiento de cada empresa fracasada con otra que no haya fracasado del mismo sector, tamaño, en función del activo total, y año, conformando así una muestra total de 60 empresas. Finalmente, se procedió a la obtención de las cuentas anuales de la muestra de empresas fracasadas y de la muestra de empresas emparejadas de los dos años previos al fracaso a fin de construir la base de datos en Excel.

\subsection{Variables consideradas}

Las variables a ser consideradas corresponden a dos grupos, uno integrado por magnitudes estructurales de la muestra y otro compuesto por una serie de ratios financieros. Para la elección de los ratios económico-financieros se analizaron varias investigaciones empíricas relevantes sobre modelos de predicción de fracaso, como así también, se hizo una revisión de la literatura de análisis contable a efectos de incluir todos aquellos ratios más frecuentemente utilizados.

Entre los estudios de predicción de insolvencia analizados se encuentran los de: Beaver (1966), Altman (1968), Pinches et al (1973), Chen y Chimerda 
(1981), Laffarga y Pina (1995), Gandía (1995), Lizarraga (1997), García (Coord.) (1997), Gallego et al (1997), Ferrando y Blanco (1998), Somoza (2000) y Henríquez (2000), y de la literatura contable fueron consultados: Lev (1978), Cañíbano (1989), Rivero y Rivero (1997), Bernstein (1993), Urías (1999), Amat (2002) y Dun and Bradstreet (2000).

En conjunto totalizaron 79 variables, siendo 6 de ellas magnitudes estructurales y 73 ratios contables. Las variables estructurales estudiadas fueron: Activo Total, Ventas, Activo Fijo, Fondos Propios, Resultado Neto y Personal. En el Cuadro 2 se exponen los ratios económico-financieros y en el Cuadro 3 se presenta la descripción de las nomenclaturas utilizadas en los ratios.

La agrupación de los ratios, mostrada en el Cuadro 2, se ha realizado, bajo un criterio simplificador, en cuatro grupos principales, por lo que los ratios de estructura del activo se encuentran dentro del grupo de ratios de Liquidez, los de estructura del pasivo dentro de los ratios de Endeudamiento y los ratios de generación de recursos han sido agrupados junto con los ratios de Rentabilidad.

\section{Cuadro 2: Ratios considerados en el estudio}

\begin{tabular}{|c|c|c|c|c|c|c|c|}
\hline $\mathrm{N}^{\circ}$ & Nombre de & Descripción & Agrup. & $\mathrm{N}^{\circ}$ & Nombre de & Descripción & Agrup. \\
\hline & la variable & & & & la variable & & \\
\hline 1 & RATIOO1 & $\mathrm{AC} / \mathrm{PC}$ & Liq. & 38 & RATIO38 & $(\mathrm{GP}+\mathrm{DA}) / \mathrm{VA}$ & Rent. \\
\hline 2 & RATIOO2 & $\mathrm{AD} / \mathrm{PC}$ & Liq. & 39 & RATIO39 & GP/V & Rent. \\
\hline 3 & RATIOO3 & $(\mathrm{T}+\mathrm{IFT}) / \mathrm{PC}$ & Liq. & 40 & RATIO40 & $\mathrm{RF} / \mathrm{V}$ & Rent. \\
\hline 4 & RATIOO4 & $\mathrm{T} / \mathrm{PC}$ & Liq. & 41 & RATIO41 & V/AT & Rot. \\
\hline 5 & RATIO05 & $\mathrm{AD} / \mathrm{AC}$ & Liq. & 42 & RATIO42 & V/AF & Rot \\
\hline 6 & RATIO06 & $\mathrm{T} / \mathrm{AC}$ & Liq. & 43 & RATIO43 & V/FP & Rot \\
\hline 7 & RATIO07 & INTÉRV.S/CRÉD. & Liq. & 44 & RATIO44 & V/DT & Rot \\
\hline 8 & RATIO08 & $(\mathrm{AC}-\mathrm{PC}-\mathrm{E}) / \mathrm{APR}$ & Liq. & 45 & RATIO45 & V/PC & Rot \\
\hline 9 & RATIO09 & $(\mathrm{T}+\mathrm{IFT}) / \mathrm{CC}$ & Liq. & 46 & RATIO46 & $\mathrm{AC} / \mathrm{V}$ & Rot \\
\hline 10 & RATIO10 & $\mathrm{CC} /(\mathrm{V}+\mathrm{OING}+\mathrm{RF})$ & Liq. & 47 & RATIO47 & $\mathrm{CC} / \mathrm{V}$ & Rot \\
\hline 11 & RATIO11 & $\mathrm{AC} / \mathrm{AT}$ & Liq. & 48 & RATIO48 & $\mathrm{AD} / \mathrm{V}$ & Rot \\
\hline 12 & RATIO12 & $\mathrm{AD} / \mathrm{AT}$ & Liq. & 49 & RATIO49 & $\mathrm{E} / \mathrm{V}$ & Rot \\
\hline 13 & RATIO13 & $(\mathrm{T}+\mathrm{IFT}) / \mathrm{AT}$ & Liq. & 50 & RATIO50 & $\mathrm{D} / \mathrm{V}$ & Rot \\
\hline 14 & RATIO14 & T/AT & Liq. & 51 & RATIO51 & $T / V$ & Rot \\
\hline 15 & RATIO15 & $\mathrm{CC} / \mathrm{AT}$ & Liq. & 52 & RATIO52 & $\mathrm{PC} / \mathrm{DT}$ & End. \\
\hline 16 & RATIO16 & RN/AT & Rent. & 53 & RATIO53 & $\mathrm{FP} / \mathrm{AT}$ & End. \\
\hline 17 & RATIO17 & $\mathrm{RAT} / \mathrm{AT}$ & Rent. & 54 & RATIO54 & FP/DT & End. \\
\hline 18 & RATIO18 & RAIT/AT & Rent. & 55 & RATIO55 & $\mathrm{PC} / \mathrm{FP}$ & End. \\
\hline 19 & RATIO19 & $\mathrm{RA} / \mathrm{AT}$ & Rent. & 56 & RATIO56 & ELP/AT & End. \\
\hline 20 & RATIO20 & RAIT y Ext./AT & Rent. & 57 & RATIO57 & PC/AT & End. \\
\hline 21 & RATIO21 & RE/AT & Rent. & 58 & RATIO58 & DT/AT & End. \\
\hline 22 & RATIO22 & VA/AT & Rent. & 59 & RATIO59 & $\mathrm{RP} / \mathrm{PT}$ & End. \\
\hline 23 & RATIO23 & CFT/AT & Rent. & 60 & RATIO60 & $\mathrm{RP} / \mathrm{PC}$ & End. \\
\hline 24 & RATIO24 & RN/FP & Rent. & 61 & RATIO61 & $\mathrm{FP} / \mathrm{AF}$ & End. \\
\hline 25 & RATIO25 & RAT/FP & Rent. & 62 & RATIO62 & $\mathrm{RP} / \mathrm{AF}$ & End. \\
\hline 26 & RATIO26 & RN/DT & Rent. & 63 & RATIO63 & AR/DT & End. \\
\hline 27 & RATIO27 & RAIT/DT & Rent. & 64 & RATIO64 & DA/IM & End. \\
\hline 28 & RATIO28 & CFT/DT & Rent. & 65 & RATIO65 & $\mathrm{DA} /(\mathrm{AF}-\mathrm{IF}+\mathrm{GDVE})$ & End. \\
\hline 29 & RATIO29 & $\mathrm{RN} / \mathrm{PC}$ & Rent. & 66 & RATIO66 & $\mathrm{BNOD} / \mathrm{AT}$ & End. \\
\hline 30 & RATIO3O & $\mathrm{RAT} / \mathrm{PC}$ & Rent. & 67 & RATIO67 & GF/VA & End. \\
\hline 31 & RATIO31 & CFT/PC & Rent. & 68 & RATIO68 & GF/V & End. \\
\hline 32 & RATIO32 & $\mathrm{RN} / \mathrm{V}$ & Rent. & 69 & RATIO69 & RAIT/GF & End. \\
\hline 33 & RATIO33 & $\mathrm{RAT} / \mathrm{V}$ & Rent. & 70 & RATIO70 & RE/GF & End. \\
\hline 34 & RATIO34 & $\mathrm{RA} / \mathrm{V}$ & Rent. & 71 & RATIO71 & VM/DT & Otros \\
\hline 35 & RATIO35 & $\mathrm{CFT} / \mathrm{V}$ & Rent. & 72 & RATIO72 & $\mathrm{CFO} / \mathrm{AT}$ & Otros \\
\hline 36 & RATIO36 & CFT/RE & Rent. & 73 & RATIO73 & $\%$ Crec.VA & Rent. \\
\hline 37 & RATIO37 & GF/DT & Rent. & & & & \\
\hline
\end{tabular}

Fonte: Elaborado por los autores. 


\section{Cuadro 3: Nomenclaturas utilizadas en los ratios}

\begin{tabular}{|c|c|}
\hline Nomenclatura & Descripción \\
\hline $\mathrm{AC}$ & Activo Circulante \\
\hline $\mathrm{AD}$ & Activos Defensivos $=\mathrm{AC}-\mathrm{E}$ \\
\hline $\mathrm{AF}$ & Activo Fijo $=$ Inmovilizado \\
\hline APROV & Aprovisionamientos \\
\hline \multirow[t]{2}{*}{ AR } & Activo Real $=$ AT - Activos Ficticios \\
\hline & Activos Ficticios $=$ GDVE + Acc.p/Desemb.No Ex. + Acc.p/Desemb.Ex. + Acc.Propias CP \\
\hline $\mathrm{AT}$ & Activo Total \\
\hline BNOD & $\begin{array}{l}\text { Beneficios No Distribuidos = Reservas = [Reservas (no R.Revaloriz. ni Prima Emis.) }+ \\
\text { Rtdos.Ej.Ant.] }\end{array}$ \\
\hline $\mathrm{CC}$ & Capital Circulante $=\mathrm{AC}-\mathrm{PC}=$ Fondo de Maniobra \\
\hline \multirow{3}{*}{ CFO } & $\begin{array}{l}\text { Cash Flow Operativo calculado a partir del CFT = CFT - Variac. AC Explot. + Variac. PC } \\
\text { Explot. }\end{array}$ \\
\hline & AC Explotación $=E+D+$ Ajustes Periodificación \\
\hline & PC Explotación = PC - Deudas con Entidades de Crédito - Emisiones Obligaciones y Otros \\
\hline CFT & Cash Flow Tradicional $=\mathrm{RN}+\mathrm{DA}+$ Provis. $\mathrm{LP}=$ Recursos Generados \\
\hline $\mathrm{D}$ & Deudores \\
\hline DA & Dotación p/Amortizaciones de Inmovilizado \\
\hline DT & Deuda Total $=$ Pasivo Exigible $=$ PT - FP $=$ Fondos Ajenos $=\mathrm{ELP}+\mathrm{PC}$ \\
\hline E & Existencias \\
\hline ELP & Exigible Largo Plazo = Acreed.a LP + Prov.Riesgos/Gtos.LP + Ing.a Dist.Vs.E. \\
\hline FP & Fondos Propios $=$ Patrimonio Neto \\
\hline GDVS & Gastos a Distribuir en Varios Ejercicios \\
\hline GF & Gastos Financieros \\
\hline IF & Inmovilizado Financiero \\
\hline IFT & Inversiones Financieras Temporales \\
\hline IM & Inmovilizado Material \\
\hline INTÉRV.S/CRÉD. & Intérvalo sin Crédito = (AC - E - PC) / (Gtos.Explot. - DA - Provis.de Tráfico $)$ \\
\hline OING & Otros Ingresos \\
\hline $\mathrm{PC}$ & Pasivo Circulante + Prov.Riesgos/Gtos.CP \\
\hline PT & Pasivo Total $=\mathrm{FP}+\mathrm{ELP}+\mathrm{PC}$ \\
\hline RA & Resultado de la Actividad = Rtdo . de las Actividades Ordinarias \\
\hline RAIT & Rtdo. antes de Int. e Imp. $=$ RAT + GF \\
\hline RAIT y Ex. & Rtdo. antes de Int., Imp.y Extraordinarios $=$ Rtdo. Actividad + GF \\
\hline RAT & Rtdo. antes de Impuestos \\
\hline $\mathrm{RE}$ & Rtdo. de la Explotación = Ingresos Exp. - Gastos Exp. \\
\hline RF & Rtdo. Financiero \\
\hline RN & Rtdo. Neto $=$ Rtdo. después de Impuestos $=$ Rtdo. del Ejercicio \\
\hline $\mathrm{RP}$ & Recursos Permanentes $=$ Pasivo Fijo $=$ FP + ELP \\
\hline $\mathrm{T}$ & Tesorería $=$ Disponible \\
\hline $\mathrm{V}$ & Ventas $=$ Importe Neto de la Cifra de Negocios \\
\hline \multirow[t]{2}{*}{ VA } & $\begin{array}{l}\text { Valor Añadido = Ingresos Exp. - Consumos Exp. - Otros Gtos. Exp. = RBE = Rtdo. Bruto de } \\
\text { Exp. }\end{array}$ \\
\hline & Consumos Exp. $=$ Reducc. Exist. Prod. + APROV + GP \\
\hline VM & $\begin{array}{l}\text { Valor de Mercado. Cap. Social = Valor Bursátil de la Empresa = Valor de la Acción a FCE por } \\
\text { cant. Acciones }\end{array}$ \\
\hline
\end{tabular}

Fonte: Elaborado por los autores.

Con base en las variables presentadas, se muestra a seguir los resultados empíricos encontrados.

\section{Resultados}

Del análisis descriptivo de las variables estructurales que caracterizan a la muestra de empresas fracasadas y sanas surgen rasgos diferenciales entre ambos grupos de empresas, considerando los dos años observados, conforme muestra 
el Cuadro 4.

Cuadro 4: Características estructurales diferenciales

\begin{tabular}{|l|c|c|}
\hline \multicolumn{1}{|c|}{ Variables estructurales } & Fracasada & Sana \\
\hline Activo Total & Mayor & Menor \\
\hline Ventas & Menor & Mayor \\
\hline Activo Fijo & Mayor & Menor \\
\hline Fondos Propios & Menor $*$ & Mayor* \\
\hline Resultado Neto & Menor & Mayor \\
\hline Personal & Menor* & Mayor* \\
\hline
\end{tabular}

Fonte: Elaborado por los autores.

* Si bien la media arrojaba el resultado contrario al presentado, se ha optado por considerar el presentado por la mediana, ya que ante la presencia de valores atípicos, esta última es más representativa que la media.

Ya con relación a los ratios contables, se desprende del análisis descriptivo la existencia de diferencias entre las medias de los ratios de los dos colectivos, observándose que las empresas fracasadas presentan valores menores en los ratios de liquidez, rentabilidad y rotación y superiores en los de endeudamiento, lo que permite realizar una primera aproximación sobre los rasgos económico-financieros que caracterizan en forma diferente a ambos grupos de empresas, que serán objeto de contraste estadístico a través de técnicas univariantes.

Para conocer si existen diferencias significativas en las medias de los ratios de las empresas fracasadas y las sanas, se realizó en primer lugar la prueba paramétrica de análisis de la varianza, conocido como ANOVA de un factor.

El resultado arrojó un total de 40 ratios, presentados en el Cuadro 5, sobre los 73 estudiados, distribuidos en las cuatro categorías en que los hemos clasificado: liquidez, rentabilidad, rotación y endeudamiento, que rechazan la hipótesis nula de igualdad de medias, siendo éstos justamente los que demostraron no rechazar la normalidad según la prueba no paramétrica de Kolmogorov-Smirnov para una muestra, test que resultó menos estricto que los paramétricos.

Cuadro 5: Ratios con diferentes medias según prueba paramétrica ANOVA

\begin{tabular}{|c|c|c|c|c|c|c|c|}
\hline $\mathbf{N}^{\mathbf{2}}$ & $\begin{array}{c}\text { Nombre de } \\
\text { la Variable }\end{array}$ & $\mathbf{N}^{\mathbf{0}}$ & $\begin{array}{c}\text { Nombre de } \\
\text { la Variable }\end{array}$ & $\mathbf{N}^{\mathbf{2}}$ & $\begin{array}{c}\text { Nombre de } \\
\text { la Variable }\end{array}$ & $\mathbf{N}^{\mathbf{0}}$ & $\begin{array}{c}\text { Nombre de } \\
\text { la Variable }\end{array}$ \\
\hline 1 & $\mathrm{R} 01$ & 11 & $\mathrm{R} 18$ & 21 & $\mathrm{R} 30$ & 31 & $\mathrm{R} 57$ \\
\hline 2 & $\mathrm{R} 02$ & 12 & $\mathrm{R} 19$ & 22 & $\mathrm{R} 31$ & 32 & $\mathrm{R} 58$ \\
\hline 3 & $\mathrm{R} 04$ & 13 & $\mathrm{R} 20$ & 23 & $\mathrm{R} 37$ & 33 & $\mathrm{R} 59$ \\
\hline 4 & $\mathrm{R} 06$ & 14 & $\mathrm{R} 21$ & 24 & $\mathrm{R} 41$ & 34 & $\mathrm{R} 61$ \\
\hline 5 & $\mathrm{R} 12$ & 15 & $\mathrm{R} 22$ & 25 & $\mathrm{R} 42$ & 35 & $\mathrm{R} 62$ \\
\hline 6 & $\mathrm{R} 13$ & 16 & $\mathrm{R} 23$ & 26 & $\mathrm{R} 44$ & 36 & $\mathrm{R} 63$ \\
\hline 7 & $\mathrm{R} 14$ & 17 & $\mathrm{R} 26$ & 27 & $\mathrm{R} 45$ & 37 & $\mathrm{R} 66$ \\
\hline 8 & $\mathrm{R} 15$ & 18 & $\mathrm{R} 27$ & 28 & $\mathrm{R} 53$ & 38 & $\mathrm{R} 69$ \\
\hline 9 & $\mathrm{R} 16$ & 19 & $\mathrm{R} 28$ & 29 & $\mathrm{R} 54$ & 39 & $\mathrm{R} 70$ \\
\hline 10 & $\mathrm{R} 17$ & 20 & $\mathrm{R} 29$ & 30 & $\mathrm{R} 56$ & 40 & $\mathrm{R} 71$ \\
\hline
\end{tabular}

Fonte: Elaborado por los autores. 
Luego se aplicó el Análisis de Perfiles a los ratios que mostraron diferencias significativas en las medias resultantes de la prueba ANOVA.. Los gráficos del análisis de perfiles de cada ratio muestran en relación a las cuatro categorías en que hemos clasificado los ratios:

A) LIQUIDEZ: Todos los ratios de liquidez (R01, R02, R04, R06, R12, $\mathrm{R} 13, \mathrm{R} 14$ y R15) muestran que las empresas fracasadas presentan menor liquidez que las sanas a lo largo del período estudiado, empeorando levemente la misma cuando se acerca la fecha de fracaso. Por su parte la evolución de los ratios de liquidez en las empresas sanas se manifiesta en términos generales bastante constante, si bien puede observarse que los ratios R01, R02, R04, R06 y R15 exhiben una tendencia constante o ascendente y los ratios R12, R13 y R14 presentan una leve tendencia a la baja.

B) RENTABILIDAD: Al igual como ocurre con los ratios de liquidez, la totalidad de los ratios de rentabilidad (R16, R17, R18, R19, R29, R21, R22, R23, R26, R27, 28, R29, R30, R31 y R37) revelan menor rentabilidad en las empresas fracasadas y un caída bastante acentuada de ésta al acercarse la fecha de fracaso, en cuanto, las empresas sanas se mantienen constantes en los dos años o con una muy leve caída. Merece comentar el ratio R37, de gastos financieros a deuda total, en donde se percibe nítidamente el camino opuesto que toman ambos grupos de empresas respecto al mismo, el cual disminuye en las sanas al pasar del segundo año anterior al fracaso al primer año previo y aumenta en las fracasadas.

C) ROTACIÓN: Los ratios de rotación (R41, R42, R44 y R45) exhiben menor rotación en las empresas fracasadas, con una muy leve tendencia a la baja en ambos grupos de empresas.

D) ENDEUDAMIENTO: Los ratios de endeudamiento (R53, R54, R56, R57, R58, R59, R61, R62, R63, R68, R69, R70 y R71) denotan en todos ellos mayores niveles de endeudamiento en las empresas fracasadas que en las sanas. Además, en la mayoría de los ratios, las empresas fracasadas presentan un aumento del mismo al acercarse a la fecha de fracaso, en cuanto, las empresas sanas se mantienen más o menos constantes.

A seguir se presenta, a través de la Figura 1, algunos de los Gráficos de Perfiles obtenidos sobre las características económico-financieras de empresas industriales españolas cotizadas que fracasaron en el periodo de 1992 a 2001. 
Figura 1: Análisis de perfiles - Gráficos de algunos de los ratios con diferentes medias según ANOVA

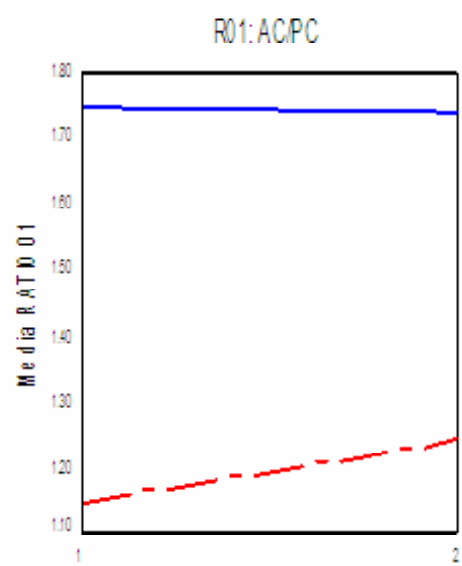

Aiosares deltacaso
R19: RA/AT

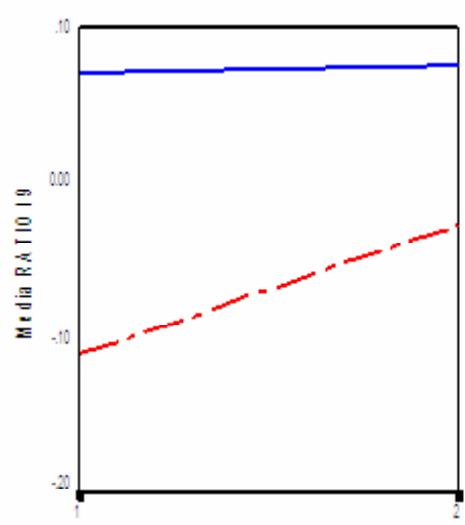

Linos anites del fracaso

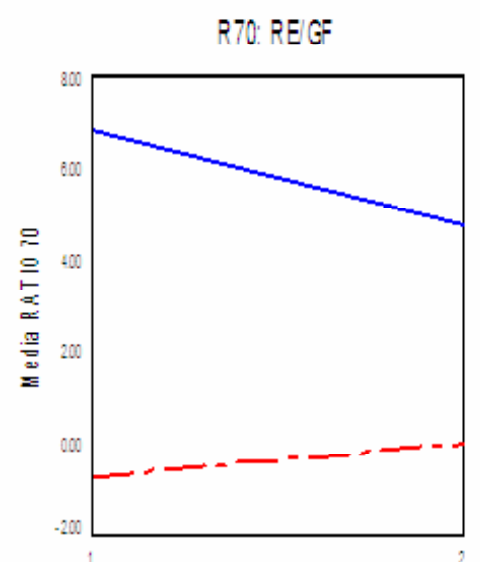

Aixs ates de iracos:

Sanas

$-\cdot \cdot \cdot$. Fracasadas

Fonte: Elaborado por los autores.

El primer gráfico es el ratio R01 de liquidez corriente, el segundo es el ratio $\mathrm{R} 19$ de rentabilidad de la actividad sobre el activo y el tercero es el ratio R70 de cobertura de gastos financieros con el resultado de la explotación.

A través da observación del gráfico de perfiles del ratio R01, de liquidez corriente, se observa que las empresas sanas presentan un nivel constante de 1,74 no periodo analizado, en cuanto las empresas fracasadas presentan un nivel decreciente, pasando de 1,24 en el segundo año anterior al fracaso para 1,14 en el año anterior.

Por su parte, el gráfico del ratio R19, de rentabilidad del activo, muestra que en media las empresas sanas tuvieron un índice estable, siendo $7,60 \%$ en el segundo año anterior al fracaso y 7,28\% en el año anterior, en cuanto, las empresas fracasadas presentan rentabilidad del activo negativa del $2,64 \%$ en el segundo año anterior al fracaso disminuyendo para $11,12 \%$ negativa en el año inmediatamente anterior al fracaso.

Ya el gráfico del ratio R70, de cobertura de gastos financieros, evidencia una tendencia opuesta entre ambos tipos de empresas. Las empresas sanas, además de presentar un nivel mayor de cobertura de gastos financieros con el resultado de la explotación, muestran una mejora en el periodo de dos años analizado pasando de $479 \%$ para $684 \%$, en cuanto, la media de las empresas fracasadas pasó de $1 \%$ para $71 \%$ negativa en el año inmediatamente anterior al fracaso.

Concluyendo sobre el perfil económico financiero que caracteriza a los dos colectivos estudiados, se observa que las empresas fracasadas presentan valores bastante menores en los ratios de liquidez, rentabilidad y rotación y superiores 
en los de endeudamiento, reforzando las hipótesis que surgieron del análisis descriptivo sobre los rasgos que caracterizan en forma diferente a ambos grupos de empresas, las cuales fueron contrastadas estadísticamente mediante los test de comparación de medias y visualmente observadas a través de los gráficos de perfiles.

Sin embargo, dado que el test de comparación de medias y los gráficos de análisis de perfiles sólo revelan diferencias en las medias de los valores de los ratios pero no tienen en cuenta la dispersión que puede existir en cada uno de ellos, se aplicó el Análisis Dicotómico a fin de poder extraer conclusiones sobre la capacidad discriminante de los ratios, de modo a responder la pregunta de investigación sobre cuáles son los índices contables que pueden alertar sobre el riesgo de insolvencia y cuáles son los valores críticos de esos índices en los años previos a la discontinuidad.

De los resultados del análisis dicotómico, se observa que 22 ratios alcanzan un porcentaje de acierto global superior al $85 \%$ en el año anterior al fracaso, por lo que se procedió a realizar el análisis dicotómico de dichos ratios para el segundo año anterior al fracaso. Los resultados de esos 22 ratios son presentados a través de la Tabla 1.

Del análisis de los resultados alcanzados, se desprende que los ratios R19 y R70 son los que proporcionan mejores resultados a nivel univariante. Ambos arrojan un porcentaje de acierto global de $95 \%$ para el año inmediato anterior al fracaso y de $83 \%$ para el segundo año anterior. Luego se encuentran los ratios R27 y R34, con un porcentaje de acierto global de 93\% para el año anterior al fracaso y de $83 \%$ para el segundo año anterior.

También existen otros ratios con buen poder predictivo, los cuales si bien arrojan un porcentaje menor de acierto global que los anteriormente presentados, continúan superiores al porcentaje de $90 \%$ de acierto global para el año anterior al fracaso y al $80 \%$ para el segundo año anterior. Ellos son, por orden de mejores resultados alcanzados, los ratios R69, R22, R26, R30, R20, R18, R23, R16 y R28.

Por lo expuesto, y a fin de elegir el mejor ratio que discrimine a las empresas fracasadas y sanas, se seleccionó el ratio R19 de resultado de la actividad a activo total como la mejor variable univariante, ya que arroja un porcentaje de error Tipo I menor que el R70 y, como fue comentado previamente, se considera este error más grave que el Tipo II.

Buscando elegir cuál o cuáles de los otros ratios podrían ser considerados también dentro de los mejores indicadores, se analizó las correlaciones existentes entre ellos, a fin de seleccionar aquellos que posean los mayores porcentajes de acierto y no estén altamente correlacionados, presentándose en la Tabla 2 los coeficientes de correlaciones de Pearson obtenidos. En base al análisis de las 
correlaciones entre los ratios con mejores poderes discriminantes, se eligieron como mejores modelos univariantes estimados los expuestos en la Tabla 3 , los cuales presentan baja correlación entre ellos.

Tabla 1: Resultado del análisis dicotómico de los 22 ratios con porcentaje de acierto global superior al $85 \%$ en $n-1$

\begin{tabular}{|c|c|c|c|c|c|c|c|c|c|c|}
\hline \multirow[b]{2}{*}{ Ratio } & \multicolumn{5}{|c|}{ n-1 } & \multicolumn{5}{|c|}{ n-2 } \\
\hline & $\begin{array}{l}\text { Punto } \\
\text { de corte }\end{array}$ & $\begin{array}{c}\% \\
\text { Acierto } \\
\text { global }\end{array}$ & $\begin{array}{c}\% \\
\text { Error } \\
\text { total }\end{array}$ & $\begin{array}{c}\% \\
\text { Error } \\
\text { Tipo I }\end{array}$ & $\begin{array}{c}\text { \% } \\
\text { Error } \\
\text { Tipo } \\
\text { II }\end{array}$ & $\begin{array}{l}\text { Punto } \\
\text { de corte }\end{array}$ & $\begin{array}{c}\% \\
\text { Acierto } \\
\text { global }\end{array}$ & $\begin{array}{c}\% \\
\text { Error } \\
\text { total }\end{array}$ & $\begin{array}{c}\% \\
\text { Error } \\
\text { Tipo I }\end{array}$ & $\begin{array}{c}\% \% \\
\text { Error } \\
\text { Tipo } \\
\text { II }\end{array}$ \\
\hline $\mathrm{R} 16$ & 0,0067572 & 91,67 & 8,33 & 6,67 & 10,00 & 0,0191722 & 80,00 & 20,00 & 26,67 & 13,33 \\
\hline $\mathrm{R} 17$ & 0,0023143 & 93,33 & 6,67 & 10,00 & 3,33 & 0,0301615 & 78,33 & 21,67 & 20,00 & 23,33 \\
\hline $\mathrm{R} 18$ & $-0,0353903$ & 91,67 & 8,33 & 6,67 & 10,00 & $-0,0220498$ & 83,33 & 16,67 & 26,67 & 6,67 \\
\hline R19 & 0,0043374 & 95,00 & 5,00 & 3,33 & 6,67 & 0,0316548 & 83,33 & 16,67 & 10,00 & 23,33 \\
\hline $\mathrm{R} 20$ & $-0,0351139$ & 91,67 & 8,33 & 6,67 & 10,00 & $-0,0353892$ & 85,00 & 15,00 & 23,33 & 6,67 \\
\hline $\mathrm{R} 21$ & 0,0359873 & 93,33 & 6,67 & 3,33 & 10,00 & 0,0448357 & 78,33 & 21,67 & 23,33 & 20,00 \\
\hline $\mathrm{R} 22$ & 0,07674 & 93,33 & 6,67 & 3,33 & 10,00 & 0,0695529 & 80,00 & 20,00 & 23,33 & 16,67 \\
\hline $\mathrm{R} 23$ & 0,0427284 & 91,67 & 8,33 & 10,00 & 6,67 & 0,037219 & 83,33 & 16,67 & 23,33 & 10,00 \\
\hline $\mathrm{R} 24$ & 0,0305822 & 88,33 & 11,67 & 13,33 & 10,00 & 0,0990434 & 76,67 & 23,33 & 13,33 & 33,33 \\
\hline $\mathrm{R} 25$ & 0,0286594 & 88,33 & 11,67 & 13,33 & 10,00 & 0,0686492 & 75,00 & 25,00 & 30,00 & 20,00 \\
\hline R26 & 0,0090665 & 93,33 & 6,67 & 6,67 & 6,67 & 0,0418872 & 80,00 & 20,00 & 20,00 & 20,00 \\
\hline $\mathrm{R} 27$ & $-0,0602472$ & 93,33 & 6,67 & 3,33 & 10,00 & $-0,0477247$ & 83,33 & 16,67 & 30,00 & 3,33 \\
\hline $\mathrm{R} 28$ & 0,0579604 & 90,00 & 10,00 & 13,33 & 6,67 & 0,0500395 & 81,67 & 18,33 & 33,33 & 3,33 \\
\hline R29 & 0,0166338 & 93,33 & 6,67 & 6,67 & 6,67 & 0,0262907 & 78,33 & 21,67 & 33,33 & 10,00 \\
\hline R30 & 0,0037827 & 93,33 & 6,67 & 10,00 & 3,33 & 0,0619831 & 80,00 & 20,00 & 23,33 & 16,67 \\
\hline $\mathrm{R} 31$ & 0,0710127 & 88,33 & 11,67 & 16,67 & 6,67 & 0,0597546 & 83,33 & 16,67 & 33,33 & 0,00 \\
\hline R32 & 0,0095817 & 93,33 & 6,67 & 6,67 & 6,67 & 0,0204891 & 78,33 & 21,67 & 30,00 & 13,33 \\
\hline R33 & 0,0078603 & 93,33 & 6,67 & 6,67 & 6,67 & 0,0410241 & 76,67 & 23,33 & 16,67 & 30,00 \\
\hline R34 & 0,0029403 & 93,33 & 6,67 & 6,67 & 6,67 & 0,0034288 & 83,33 & 16,67 & 26,67 & 6,67 \\
\hline $\mathrm{R} 35$ & 0,0432272 & 85,00 & 15,00 & 20,00 & 10,00 & 0,0423744 & 81,67 & 18,33 & 36,67 & 0,00 \\
\hline R69 & 1,1404738 & 93,33 & 6,67 & 6,67 & 6,67 & 1,8226325 & 81,67 & 18,33 & 13,33 & 23,33 \\
\hline $\mathrm{R} 70$ & 1,0152643 & 95,00 & 5,00 & 3,33 & 6,67 & 0,9929656 & 83,33 & 16,67 & 23,33 & 10,00 \\
\hline
\end{tabular}

Fonte: Elaborado por los autores.

Tabla 2: Correlaciones entre los ratios que mejor discriminan ambos grupos

\begin{tabular}{l|c|c|c|c|c|c|c|c|c|c|c|c|c}
\hline & R16 & R18 & R19 & R20 & R22 & R23 & R26 & R27 & R28 & R30 & R34 & R69 & R70 \\
\hline R16 & 1,000 & 0,985 & 0,939 & 0,918 & 0,857 & 0,965 & 0,889 & 0,854 & 0,791 & 0,664 & 0,044 & 0,386 & 0,392 \\
\hline R18 & & 1,000 & 0,962 & 0,959 & 0,869 & 0,952 & 0,900 & 0,891 & 0,814 & 0,673 & 0,064 & 0,433 & 0,445 \\
\hline R19 & & & 1,000 & 0,991 & 0,922 & 0,909 & 0,849 & 0,848 & 0,767 & 0,622 & 0,103 & 0,415 & 0,445 \\
\hline R20 & & & & 1,000 & 0,898 & 0,892 & 0,841 & 0,854 & 0,769 & 0,612 & 0,108 & 0,434 & 0,466 \\
\hline R22 & & & & & 1,000 & 0,895 & 0,796 & 0,789 & 0,763 & 0,597 & 0,180 & 0,350 & 0,384 \\
\hline R23 & & & & & & 1,000 & 0,872 & 0,840 & 0,841 & 0,654 & 0,047 & 0,373 & 0,380 \\
\hline R26 & & & & & & & 1,000 & 0,987 & 0,955 & 0,794 & 0,082 & 0,525 & 0,511 \\
\hline R27 & & & & & & & & 1,000 & 0,950 & 0,783 & 0,117 & 0,561 & 0,554 \\
\hline R28 & & & & & & & & & 1,000 & 0,764 & 0,064 & 0,497 & 0,480 \\
\hline R30 & & & & & & & & & & 1,000 & 0,074 & 0,428 & 0,411 \\
\hline R34 & & & & & & & & & & & 1,000 & 0,069 & 0,084 \\
\hline R69 & & & & & & & & & & & & 1,000 & 0,994 \\
\hline R70 & & & & & & & & & & & & & 1,000 \\
\hline
\end{tabular}

Fonte: Elaborado por los autores. 
Tabla 3: Mejores modelos univariantes estimados

\begin{tabular}{|c|c|c|c|c|c|c|c|c|c|c|c|}
\hline \multirow[b]{2}{*}{ Ratio } & \multirow[b]{2}{*}{ Descrip. } & \multicolumn{5}{|c|}{$n-1$} & \multicolumn{5}{|c|}{$n-2$} \\
\hline & & $\begin{array}{l}\text { Punto } \\
\text { de corte }\end{array}$ & $\begin{array}{c}\% \% \\
\text { Acierto } \\
\text { global }\end{array}$ & $\begin{array}{c}\% \\
\text { Error } \\
\text { total }\end{array}$ & $\begin{array}{c}\% \\
\text { Error } \\
\text { Tipo I }\end{array}$ & $\begin{array}{c}\text { \% } \\
\text { Error } \\
\text { Tipo } \\
\text { II }\end{array}$ & $\begin{array}{l}\text { Punto } \\
\text { de corte }\end{array}$ & $\begin{array}{c}\% \% \\
\text { Acierto } \\
\text { global }\end{array}$ & $\begin{array}{c}\% \\
\text { Error } \\
\text { total }\end{array}$ & $\begin{array}{c}\% \\
\text { Error } \\
\text { Tipo I }\end{array}$ & $\begin{array}{c}\% \\
\text { Error } \\
\text { Tipo } \\
\text { II }\end{array}$ \\
\hline R19 & $\mathrm{RA} / \mathrm{AT}$ & 0,004337 & 95,00 & 5,00 & 3,33 & 6,67 & 0,0316548 & 83,33 & 16,67 & 10,00 & 23,33 \\
\hline $\mathrm{R} 70$ & $\mathrm{RE} / \mathrm{GF}$ & 1,015264 & 95,00 & 5,00 & 3,33 & 6,67 & 0,9929656 & 83,33 & 16,67 & 23,33 & 10,00 \\
\hline R34 & $\mathrm{RA} / \mathrm{V}$ & 0,00294 & 93,33 & 6,67 & 6,67 & 6,67 & 0,0034288 & 83,33 & 16,67 & 26,67 & 6,67 \\
\hline
\end{tabular}

Fonte: Elaborado por los autores.

Entre los ratios seleccionados como mejores modelos univariantes se encuentra en primer lugar el ratio de rentabilidad económica R19, compuesto por el Resultado de las Actividades Ordinarias a Activo Total, luego continúa el ratio R70 de cobertura de la deuda formulado por el Resultado de la Explotación a Gastos Financieros, y como último seleccionado, el ratio R34 de margen de beneficios compuesto por el Resultado de las Actividades Ordinarias a Ventas.

Los ratios seleccionados reflejan que los indicadores que captan con altísima capacidad de acierto los síntomas claves del progresivo fracaso de una empresa son ratios de rentabilidad y de endeudamiento. Así, el ratio R19 de Rentabilidad del Activo evidencia la importancia para la continuidad de la empresa de mantener una relación adecuada entre la rentabilidad ordinaria y el activo total. Ya el segundo ratio elegido, R70 de Resultado de las Actividades Ordinarias antes del Resultado Financiero (Resultado de la Explotación) a Gastos Financieros demuestra el rol importante que juegan los malos planteamientos financieros en la insolvencia posterior de la empresa. Finalmente, el tercer ratio escogido, R34 de Margen de Beneficio del Resultado Ordinario, denota la necesidad de conquistar al menos un mínimo de margen de beneficio para mantener la salud financiera de la empresa.

\section{Comparación con resultados de otros estudios}

Beaver (1966) alcanzó con su mejor ratio de Cash flow a deuda total un porcentaje de $90 \%$ en el año previo y de $80 \%$ en el segundo año previo. Dicho ratio corresponde en nuestro estudio al ratio $\mathrm{R} 28$, con el cual hemos obtenido un porcentaje de acierto del $90 \%$ para el año anterior y del $82 \%$ para el segundo año anterior, con lo cual han sido tan buenos cuanto los de Beaver (1966).

Deakin (1972) obtuvo un porcentaje de 80\% para el año previo con el mejor ratio de Beaver (1966), el cual hemos superado en nuestro estudio, y Lizarraga (1995) alcanzó un porcentaje de 90\% para el año previo también con dicho ratio de Beaver (1966) que se corresponde con el obtenido en la presente investigación.

Los resultados de las pruebas de clasificación dicotómica de Lizarraga 
(1997) mostraron, en forma semejante a nuestro estudio, alta eficiencia de los ratios de rentabilidad de activos, con porcentajes superiores al $80 \%$, en cuanto nuestros resultados arrojaron porcentajes superiores al 90\%, disminuyendo cuando la rentabilidad de activos es calculada sin tener en cuenta el efecto de la carga financiera. El ratio de endeudamiento, R70, de resultado de la explotación a gastos financieros también produjo buenos resultados, con un porcentaje de acierto del $75 \%$, siendo que ese ratio fue uno de los mejores en el acierto de clasificación en nuestro estudio, alcanzando el porcentaje de 95\%.

En el estudio de García et al (1998) el mejor resultado alcanzado fue con el ratio de rentabilidad financiera de los recursos totales, $\mathrm{R} 17$ de resultado antes de impuestos a pasivo total, con un porcentaje de acierto global de $71 \%$ y de 69\%, para el primer y segundo año anterior al fracaso, respectivamente. Ya en nuestro estudio ese ratio arrojó un porcentaje de acierto del 93\% y 78\%.

Por tanto, merece ser destacado que los resultados conseguidos a nivel univariante en este estudio empírico muestran porcentajes de acierto mayores a los comentados sobre estudios previos y no sólo para el mejor ratio de Beaver (1966), ya que otros ratios superan inclusive el porcentaje obtenido por el de Cash flow a deuda total. Así lo demuestran los resultados de la clasificación dicotómica de los ratios R19, R70, R27, R34, R69, R22, R26, R30, R20, R18, R23 y R16, que superan el porcentaje de $90 \%$ de acierto global en el año anterior al fracaso.

\section{Conclusiones}

La pregunta de investigación de este trabajo fue conocer cuáles son los índices contables que pueden alertar sobre el riesgo de insolvencia y en que niveles esos índices indicarían riesgo de discontinuidad. En función de esa problemática el objetivo propuesto en este estudio fue elaborar modelos univariantes de previsión de insolvencia de empresas españolas industriales y de gran dimensión.

El análisis descriptivo de las muestras exhibió que las variables estructurales ventas, resultado neto, fondos propios y número de empleados son menores en las fracasadas y la variable activo fijo mayor. Ya con relación a la evolución del segundo año anterior al fracaso respecto al primero las variables activo total, ventas, fondos propios y resultado neto disminuyeron en las fracasadas y aumentaron en las sanas. La variable activo fijo aumentó en ambos colectivos y el número de empleados se mantuvo bastante estable.

La comparación de las medias de los ratios de las empresas fracasadas y sanas permitió tener una primera aproximación sobre las características económico-financieras de cada grupo, cuya significatividad estadística fue verificada luego a través del análisis de la varianza de un factor, que mostró que en la mayoría de los ratios se rechaza la hipótesis nula de igualdad de medias entre las empresas 
fracasadas y las empresas sanas.

Los gráficos del análisis de perfiles de Beaver, aplicados a los ratios con diferencias significativas en las medias de cada grupo, mostraron que todos los ratios de cada tipo presentan el mismo comportamiento, tanto con relación al nivel entre ambos grupos de empresas como con relación a su evolución. Así, los ratios de liquidez y rentabilidad son menores en las empresas fracasadas que en las sanas, empeorando, principalmente los ratios de rentabilidad, en las fracasadas al acercarse la fecha de fracaso, en cuanto en las sanas se mantienen constantes. Los ratios de rotación también son menores en las fracasadas y su tendencia en el período de dos años analizado fue en ambos grupos de empresas a la baja. Por último, los ratios de endeudamiento muestran la situación contraria, son mayores en las empresas fracasadas y aumentaron al acercarse la fecha de fracaso, en cuanto en las sanas se mantuvieron constantes.

Finalmente, el test de clasificación dicotómica aplicado proporcionó como mejores modelos univariantes tres ratios, no correlacionados entre ellos, siendo estos: Rentabilidad de la Inversión, definido como resultado de la actividad a activo total, Cobertura de Gastos Financieros con Resultados de la Explotación y Margen de Beneficio de los Resultados de la Actividad, los cuales arrojan excelentes resultados de clasificación, con acierto global en el año anterior al fracaso del 95\% y en el segundo año anterior del $83 \%$, resultando superiores a los verificados en estudios previos.

Esto demuestra que modelos elaborados con una única variable, donde su estimación y, principalmente, aplicación resulta mucho más fácil que los modelos multivariantes, constituyen una excelente herramienta para la predicción de insolvencia.

De esta forma, este estudio cumplió con su objetivo en la medida que presentó las diferencias económico-financieras de las empresas españolas cotizadas fracasadas y solventes, aportando modelos univariantes que permiten clasificar, con un alto grado de acierto, ambos grupos de empresas, permitiendo realizar análisis sobre las posibles causas que llevaron al fracaso de las empresas estudiadas.

El estudio presentado limita su objetivo a una primera etapa de estimación de modelos que permitan detectar relaciones estadísticamente significativas entre determinados indicadores económico-financieros y la insolvencia empresarial. En una segunda etapa, a partir de la obtención de nuevas muestras de empresas y datos contables de períodos posteriores, se podrá completar el presente estudio contrastando el poder de predicción de los modelos estimados a fin de proporcionar herramientas útiles para la toma de decisiones por los usuarios de los estados contables.

\section{Referencias}


ALTMAN, E. et al. Zeta Analysis, Journal of Banking and Finance, p. 29-54, Jun., 1977.

ALTMAN, E. Financial Ratios, Discriminant Analysis and The Prediction of Corporate Bankruptcy, Journal of Finance, p. 589-609, Sept., 1968.

AMAT, O. Análisis Económico Financiero, 18. ed., Madrid: Gestión 2000, 2002.

BEAVER, W. H. Financial Ratios as Predictors of Failure, in Empirical Research in Accounting Selected Studies, in supplement to the Journal of Accounting Research, v. 4, p. 71-111, Jan., 1966.

BERNSTEIN, L. Análisis de Estados Financieros, Barcelona: Irwin, 1993.

BLUM, M. Failing Company Discriminant Analysis, Journal of Accounting Research, p. 1-25, spring, 1974.

CAÑIBANO, L. Contabilidad: Análisis Contable de la Realidad Económica. Madrid: Pirámide, 1989.

CHEN, K., SHIMERDA, T. An Empirical Analysis of Useful Financial Ratios, Financial Management, v. 10, n. 1, p. 51-60, spring, 1981.

DEAKIN, D. A Discriminant Analysis as Predictor of Business Failure, Journal of Accounting Research, p. 167-179, spring, 1972.

DUN AND BRADSTREET. Libro de Normas y Ratios Financieros, Madrid: Dun \& Bradstreet, 2000.

EDMISTER, R. O. An Empirical Test of Financial Ratios Analysis for Small Business Failure Prediction, Journal of Financial and Quantitative Analysis, p. 1477-1493, Mar., 1972.

ELAM, R. The Effect of Lease Data on the Predictive Ability of Financial Ratios. The Accounting Review, p. 25-43, Jan., 1975.

FERRANDO, M., BLANCO, F. La Previsión del Fracaso Empresarial en la Comunidad Valenciana: Aplicación de los Modelos Discriminante y Logit, Revista Española de Financiación y Contabilidad, v. XXVII, n. 95, p. 499-540, abr.jun., 1998.

GABÁS, F. Predicción de la Insolvencia Empresarial, en CALVO-FLORES, A., GARCÍA, D. (Coord.). Predicción de la Insolvencia Empresarial. Madrid: 
AECA, p. 11-31, 1997.

GALLEGO, A. M. et al. Modelos de Predicción de Quiebras en Empresas No Financieras, Actualidad Financiera, n. 5, p. 3-14, mayo, 1997.

GANDÍA, J. L. et al. Estudio Empírico de la Solvencia Empresarial en la Economía Valenciana, Instituto Valenciano de Investigaciones Económicas, WP-EC-95-05, 1995.

GARCÍA, D. (Coord.). El Riesgo Financiero de la Pequeña y Mediana Empresa en Europa, Madrid: Pirámide, 1997.

GARCIA, D. et al. El Riesgo de Insolvencia en la Industria Manufacturera, Boletín de AECA, n. 45, p. 31-34, nov. 97-feb. 98, 1998.

GENTRY, J. et al. Classifying Bankrupt Firms with Funds Flow Components, Journal of Accounting Research, v. 23, n. 1, p. 146-160, spring, 1985.

HENRÍQUEZ, N. Fracaso Empresarial: Líneas Actuales de Investigación, Barcelona: Tesis Doctoral: Dpto. de Contab., Universidad de Barcelona, 2000.

LAFFARGA, J., PINA, V. La Utilidad del Análisis Multivariante para Evaluar la Gestión Continuada de las Empresas, Revista Española de Financiación y Contabilidad, v. XXIV, n. 84, p. 727-748, abr.-jun., 1995.

LEV, B. Análisis de los Estados Financieros: Un Nuevo Enfoque. Madrid: ESIC, 1978.

LEV, B. Decomposition Measures for Financial Analysis, Financial Management, p. 56-63, spring, 1973.

LIZARRAGA, F. Información Contable y Fracaso Empresarial: Una Contrastación de los Resultados de Beaver con Datos del Registro Mercantil, VIII Congreso de la Asociación Española de Contabilidad y Administración de Empresas (AECA), Internacionalización de la Empresa: Un Desafío para el 2000. Madrid: AECA, t. I, p. 601-618, 1995.

LIZARRAGA, F. Utilidad de la Información Contable en el Proceso de Fracaso: Análisis del Sector Industrial de la Mediana Enpresa Española, Revista Española de Financiación y Contabilidad, n. 92, p. 873-915, 1997.

MARTÍN, J. L. Modelos de Pronóstico de la Insolvencia Empresarial, en CALVOFLORES, A., GARCÍA, D. (Coord.). Predicción de la insolvencia empresarial. Madrid: AECA, p. 33-49, 1997. 
MOYER, R. C. Forecasting Financial Failure: A Reexamination, Financial Management, p. 11-17, spring, 1977.

ONTIVEROS, E., VALERO, F. Crisis Económica y Crisis Empresarial, en Crisis Económica y Derecho Concursal. Madrid: Consejo General de Colegios de Economistas de España, p. 13-32, 1987.

ONUSIC, L. M., CASA NOVA, S. P. C. A Utilização Conjunta das Técnicas Análise por Envoltória de Dados e Regressão Logística no Estudo de Insolvência de Empresas: um estudo exploratório. In: ENANPAD, XXX, 2006. Salvador. Anais... Salvador-BA: ANPAD, 2006. CD-ROM.

PINCHES, G. E., et al. The Stability of Financial Patterns in Industrial Organizations, Journal of Finance, v. 28, n. 2, p. 389-396, May, 1973.

RIVERO, J., RIVERO, M. J. Análisis de Estados Financieros, Madrid: Trivium, 1997.

SOMOZA, A. Los Modelos Contable-Financieros de Predicción de la Insolvencia Empresarial. Una Aportación y su Aplicación a una Muestra de Empresas de los Sectores Textil y Confección de la Provincia de Barcelona (1994-1997). v. I y II. Barcelona, Tesis Doctoral: Dpto. de Contab., Fac. Cs. Econ. y Emp., Universidad de Barcelona, 2000.

TAFFLER, R, TISSHAW, H. Going, Going, Gone-Four, Accountancy, n. 1003, p. 50-54, Mar., 1977.

TAFFLER, R. Forecasting Company Failure in the UK Using Discriminant Analysis and Finance Ratio Data, Journal of the Royal Statistical Society, s. A3, n. 145, pte. 3, p. 342-358, 1982.

URÍAS, J. Análisis de Estados Financieros, 2. ed., Madrid: McGraw-Hill, 1999.

WILCOX, J. W. A Prediction of Business Failure Using Accounting Data, Empirical Research in Accounting Selected Studies, in Supplement of the Journal of Accounting Research, p. 163-179, 1973. 